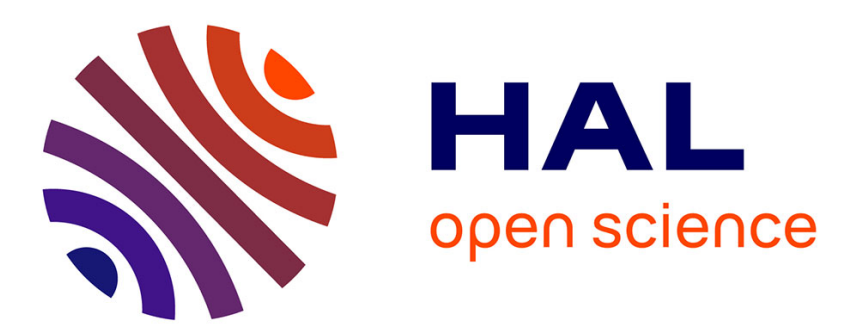

\title{
Measurement-based Analysis of the Effect of Duty Cycle in IEEE 802.15.4 MAC Performance
}

Francois Despaux, Ye-Qiong Song, Abdelkader Lahmadi

\section{To cite this version:}

Francois Despaux, Ye-Qiong Song, Abdelkader Lahmadi. Measurement-based Analysis of the Effect of Duty Cycle in IEEE 802.15.4 MAC Performance. CSCPS - 1st International Workshop on Compressive Sensing in Cyber-Physical Systems - 2013, Oct 2013, Hangzhou, China. hal-00877466

\section{HAL Id: hal-00877466 https://hal.inria.fr/hal-00877466}

Submitted on 28 Oct 2013

HAL is a multi-disciplinary open access archive for the deposit and dissemination of scientific research documents, whether they are published or not. The documents may come from teaching and research institutions in France or abroad, or from public or private research centers.
L'archive ouverte pluridisciplinaire HAL, est destinée au dépôt et à la diffusion de documents scientifiques de niveau recherche, publiés ou non, émanant des établissements d'enseignement et de recherche français ou étrangers, des laboratoires publics ou privés. 


\section{Measurement-based Analysis of the Effect of Duty Cycle in IEEE 802.15.4 MAC Performance}

\author{
François Despaux \\ LORIA, Université de Lorraine \\ Vandoeuvre-lès-Nancy, 54500, France \\ Email: francois.despaux@loria.fr
}

\author{
Ye-Qiong Song \\ LORIA, Université de Lorraine \\ Vandoeuvre-lès-Nancy, 54500, France \\ Email: song@loria.fr
}

\author{
Abdelkader Lahmadi \\ LORIA, Université de Lorraine \\ Vandoeuvre-lès-Nancy, 54500, France \\ Email: abdelkader.lahmadi@loria.fr
}

\begin{abstract}
IEEE 802.15.4 protocol stack is the basis of many wireless sensor networks (WSN) and has been proposed for low data rate and low power applications. The standard defines a duty cycle in order to allow devices to achieve efficient energy consumption. Defining the best duty cycle configuration becomes important to extend the network life time. Several works have been done in order to study the behavior of the protocol when considering duty cycle configuration and how this configuration impacts its performance parameters. Usually, the analysis is evaluated by using simulation tools. The objective of this paper is to bring an analysis of the IEEE 802.15.4 duty cycle when considering a real scenario over TinyOS and Telosb motes instead of using a simulation approach. We show through measurement how duty cycle impacts in performance metrics such as average delay and packet drop rate in realistic scenarios.
\end{abstract}

Keywords-TinyOS implementation; MAC Protocols; Duty Cycle; Wireless Sensor Networks.

\section{INTRODUCTION}

IEEE 802.15.4 protocol stack [1] is the basis of many wireless sensor networks (WSN) and has been proposed for low data rate and low power applications. Understanding the behavior and performance limitation of the protocol is challenging. WSN consist of spartially distributed autonomous sensor or devices to monitor pysical or environmental events. To facilitate network deployment, most devices are unplugged and operated by batteries. A WSN is built of nodes from a few to several hundred or even thousand and changing device batteries becomes difficult and sometimes impossible to carry out. Then, how to achieve efficient energy consumption in this kind of network is critical. IEEE 802.15.4 has two operation modes: non-beacon enabled and beacon enabled. When operating in beaconenabled mode a superframe consisting of a contention access period (CAP), contention free period (CFP) and an inactive period is periodically disseminated by the PAN coordinator and delimited by successive beacon frames. Any associated devices are allowed to communicate in the active period and conserve energy by turning off their transceivers during the inactive period. The length of the beacon interval

\footnotetext{
${ }^{0}$ This work was partially supported by Quasimodo project under No. ANR 2010 INTB 020601 in France and No. NSFC 61061130563 in China
}

and active period are specified by two system parameters, maxBeaconOrder $(\mathrm{BO})$ and macSuperframeOrder (SO). Different duty cycle configurations (and then efficient energy consumption) can be achieved by varying both $B O$ and $S O$. Besides, a specific duty cycle can be achieved by setting several different $(B O, S O)$ pairs and for each of these pairs, a different impact on throughput, packet drop rate, delay, etc. will be also achieved. In [3], authors analyse IEEE 802.15.4 duty cycle operation. Performance metrics, including queuing drop rate, goodput, and power consumption, are evaluated using a proposed analytical model and NS2 based simulation. Neugebauer et al. [4] proposed a new algorithm for $B O$ adaptation in IEEE 802.15.4 and analyzed the performance using their own developed simulator. Shu et al. [5] implemented a C-based simulation model for IEEE 802.15.4 MAC to determine optimal $B O$ and $S O$ values such that overall network energy consumption is minimized. However, results in all of these works were validated by simulation and not by considering a real environment with real motes. We are interested in analysing the protocol in real scenarios (real nodes) so that real phenomena such as capture effect, collisions, interferences among others issues can be taken into account. We have shown in [2] some issues that may arise when considering real scenarios, specially how delay is affected by realistic conditions such as the underlying operating system running in real nodes. Besides, most of the existing works focalize their attention in studying the performance parameter when varying the duty cycle configuration $(12,5 \%, 25 \%, 50, \%$, etc.) instead of analysing the performance parameters when varying both $(B O, S O)$ for a fixed duty cycle. In [3], authors show results of packet drop rate for different buffer sizes when varying the superframe size for a given duty cycle configuration. Our contribution in this paper is to bring an analysis of the IEEE 802.15.4 duty cycle when considering a real scenario over TinyOS and Telosb motes instead of using a simulation approach. In concrete, to analyse how duty cycle impacts in the delay for successful packet sent and packet drop rate when considering different configurations of $(B O, S O)$ pairs, differents packet arrival rate, buffer size and duty cycle configurations. Specially, we are interested 
in how performance parameters are impacted when varying $(B O, S O)$ for a specific duty cycle configuration. The TKN154 MAC implementation over TinyOS used in this paper is explained in [2]. We consider the delay from the moment a packet is put in the MAC buffer until the reception of the corresponding acknowledgement. We present results in terms of the delay and packet drop rate.

This paper is organized as follows. In Section II we present an overview of the IEEE 802.15.4 MAC protocol and its Duty Cycle Configuration. Experiments and Results are presented in Section III and IV respectively. A discussion regarding the results is given in Section V and we conclude our work in Section VI.

\section{OVERVIEW OF IEEE 802.15.4 STANDARD}

The IEEE 802.15.4 standard defines the PHY and MAC sublayer specifications for low-rate WPANs (LR-WPANs). The main idea of the MAC sub-layer in the IEEE 802.15.4 protocol is to provide an interface between the PHY layer and the higher layer protocols of LR-WPANs. Like the IEEE 802.11 protocol, the standard make use of CSMA/CA as the channel access protocol and it also brings support for contention-free and contention-based periods. Two operational modes are supported, beacon enabled and Non beacon-enabled modes. In beacon enabled mode a superframe structure is proposed in order to manage the communication between devices. The superframe format is defined by the PAN coordinator and is sent to the other devices within each beacon frame. The superframe structure is shown in Figure 1. It is bounded by two consecutive beacon frames and includes one Contention Access Period (CAP) and optionally a Contention Free Period (CFP). If no CFP is specified, devices that wish to communicate within the CAP period must compete with the others by means of a slotted CSMA/CA mechanism. All transmissions must be finished before the end of the superframe, i.e. before the beginning of the inactive period. The transmission is started if and only if the remaining number of backoff periods in the current superframe is sufficient to handle both the frame and the subsequent acknowledgement transmissions. Otherwise, transmission is deferred until the next superframe. In case that CFP is specified (due to some QoS requirements), Guaranteed Time Slots (GTSs) must be allocated by the PAN coordinator in order to meet low-latency or specific data bandwidth requirements. CFP is also shown in Figure 1. In both configuration the superframe structure can have an inactive period during which devices may enter in a low power mode to save energy. As seen in Figure 1, the superframe structure defines an active and an inactive period. The length of these periods is defined by two parameters: macBeaconOrder(BO) and macSuperframeOrder(SO). The former determines the interval at which the coordinator must transmit beacon frames. Then the beacon interval $B I$ is

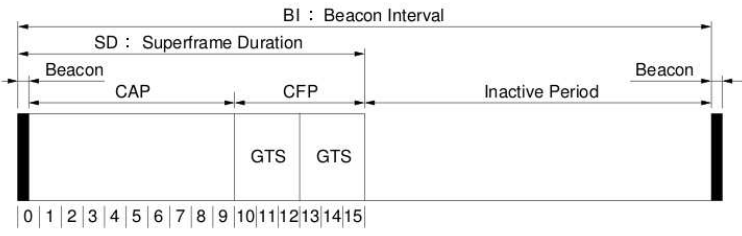

Figure 1: Superframe Structure

defined as

$$
B I=\text { aBaseSuperframeDuration } * 2^{B O}
$$

The second parameter describes the length of the active portion of the superframe. The relationship between macSuperframeOrder and the Superframe Duration $(S D)$ is

$$
S D=\text { aBaseSuperframeDuration } * 2^{S O}
$$

where aBaseSuperframeDuration = aBaseSlotDuration $*$ aNumSuperframeSlots and aNumSuperframeSlots $=16$ represents the number of equally spaced slots of the active portion of the superframe. aBaseSlotDuration represents the number of symbols (60 syms) forming a superframe slot when the superframe order is equal to zero ( 1 syms $=4$ bits). One slot contains 3 backoffs since the constant aUnitBackoffPeriod equal to 20 symbols. Finally, $B O$ and $S O$ must satisfy the constraint $0<=S O<=B O<=14$. The duty cycle is the ratio of the length of an active period $S D$ to the length of a $B I$, and is calculated as $\left(\frac{1}{2}\right)^{B O-S O}$. In this way, by handling both $S O$ and $B O$ we can get different duty cycle configuration. For instance, by setting $B O=5$ and $S O=4$ we get a duty cycle of $50 \%$ having active and inactive periods of $0.245 \mathrm{~s}$ and a beacon interval of $0.491 \mathrm{~s}$ while by setting $B O=5$ and $S O=3$ we get a $25 \%$ duty cycle where active and inactive periods are $0.122 s$ and $0.369 s$ respectively. Additionally, divers length of active and inactive periods can be obtained for a given duty cycle. For instance, by setting $B O=1$ and $S O=0$ we also obtain a $50 \%$ duty cycle but having short active and inactive periods of $0.015 \mathrm{~s}$. Different pairs under the same duty cycle have differing impacts on throughput, delay and energy efficiency.

\section{EXPERIMENTS}

Our objective in this paper is to give a comprehensive and experimental investigation on how the delay for succesful packet sent is affected when considering some parameters such as packet arrival rate, buffer size and duty cycle configuration. Specially, we study the impact in the delay and packet drop rate (which gives an idea on how the throughput is affected) when varying both $B O$ and $S O$ for a given duty cycle. As we explained before, different configurations of $B O$ and $S O$ can be set to obtain a specific duty cycle. We explore then the best configuration of $B O$ and $S O$ in terms of delay and packet drop rate for different input 
parameters. We consider a star topology with a centralized coordinator and four devices or sensor nodes are located around the coordinator, as depicted in Figure 2. Distance between devices and coordinator is the same for all scenarios and was set to 1 meter. The transmission power for each node was set to $0 \mathrm{dBm}$. We know from [6] that, for this transmission power and considering a distance of 1 meter, the packet reception rate is almost 1 since the transitional region (a region characterized by unreliable and asymetric links with high variance in reception rate) starts at a distance of almost 10 meters. Packet payload is fixed as 34 bytes for all scenarios. Channel bitrate is $250 \mathrm{kbps}$. Default values of MAC parameters are summarized in Table I. We considered

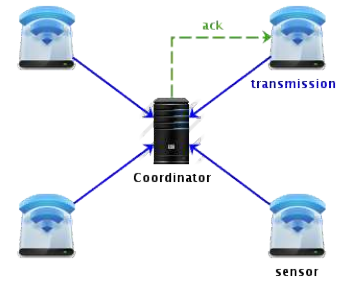

Figure 2: Star Topology Scenarios.

two different values of duty cycle: $25 \%, 50 \%$. (BO, SO) pairs for each duty cycle $\mathcal{D C}$ are specified as follows:

$$
\begin{aligned}
& \mathcal{D C}_{25}=\{(4,2),(5,3),(6,4),(7,5),(8,6),(9,7)\} \\
& \mathcal{D C}_{50}=\{(3,2),(4,3),(5,4),(6,5),(7,6),(8,7)\}
\end{aligned}
$$

The arrival rate $\lambda$ is taken from the set $\mathcal{A}=\{1,10,20,50\}$ packets per second and buffer length $l$ on each node from $\mathcal{L}=\{5,20\}$ packets. The experimentations were done as follows. For a given value of $\lambda$, buffer length $l$ and duty cycle $\mathcal{D C} \mathcal{C}_{\#}$ we took the first pair of $(B O, S O)$ for the current duty cycle and we measured the delay $d_{i}$ and packet drop rate $r_{i}$ for the current execution $i$. The duration of the execution is 3 minutes. We repeat the same experiment 10 times and we compute the average delay $\tilde{d}_{B O, S O}=\frac{1}{10} \sum_{i} d_{i}$ and average packet drop rate $\tilde{r}_{B O, S O}=\frac{1}{10} \sum_{i} r_{i}$ for the current pair $(B O, S O)$. We then repeat the whole process keeping both $\lambda$ and $l$ but moving to the next pair $(B O, S O)$ for the current duty cycle. This process is repeated until we have finished with all duty cycle pairs of $\mathcal{D C} \mathcal{C}_{\#}$. We then plot the average delays and packet drop rate for each $(B O, S O)$ found previously. The same process is repeated for all $\lambda$ values keeping the same duty cycle $\mathcal{D} \mathcal{C}_{\#}$ and buffer length $l$. Once we have finished we proceed to change the buffer length $l$ and we repeat exactly the same procedure for this new buffer length value, always considering the same duty cycle $\mathcal{D} \mathcal{C}_{\#}$. Finally, we repeat the whole process for the other duty cycles $\mathcal{D} \mathcal{C}_{\#}$. Table II shows each of the scenarios.

\begin{tabular}{|l|l|}
\hline Parameter & Value \\
\hline Max Frame Retries & 3 \\
\hline Max CSMA Backoff & 4 \\
\hline Max Backoff Exponent & 5 \\
\hline Min Backoff Exponent & 3 \\
\hline Battery Life Extension & False \\
\hline
\end{tabular}

Table I: MAC parameters on each node

\begin{tabular}{|c|c|c|c|c|}
\hline Duty Cycle & $\lambda$ (packets/second) & BO & SO & $\begin{array}{c}\text { Buffer } \\
\text { Length }\end{array}$ \\
\hline \multirow{5}{*}{$25 \%$} & $1,10,20,50$ & 4 & 2 & 5,20 \\
\cline { 2 - 5 } & $1,10,20,50$ & 5 & 3 & 5,20 \\
\cline { 2 - 5 } & $1,10,20,50$ & 6 & 4 & 5,20 \\
\cline { 2 - 5 } & $1,10,20,50$ & 7 & 5 & 5,20 \\
\cline { 2 - 5 } & $1,10,20,50$ & 8 & 6 & 5,20 \\
\hline \hline \multirow{5}{*}{$50 \%$} & $1,10,20,50$ & 9 & 7 & 5,20 \\
\cline { 2 - 5 } & $1,10,20,50$ & 3 & 2 & 5,20 \\
\cline { 2 - 5 } & $1,10,20,50$ & 4 & 3 & 5,20 \\
\cline { 2 - 5 } & $1,10,20,50$ & 5 & 4 & 5,20 \\
\cline { 2 - 5 } & $1,10,20,50$ & 6 & 5 & 5,20 \\
\cline { 2 - 5 } & $1,10,20,50$ & 7 & 6 & 5,20 \\
\hline
\end{tabular}

Table II: Scenarios

\section{RESULTS}

In this section we present the results we have obtained from the experimentations. We present results in terms of the average delay from the moment a packet arrives to the MAC buffer until the acknowledgement is received. We also present results of the packet drop rate for each of the scenarios as well as the percentage of deferred transmissions. Figures 3, 4, 5 and 6 summarize results for the packet delay and the packet drop rate for each of the scenarios. $\mathrm{X}$-axis match the ordered set of pairs $(B O, S O)$ for the corresponding duty cycle $\mathcal{D C}$ as shown in (3). Finally tables III and IV show the percentages of deferred transmissions for each scenario. In the next section we present a discussion of the results.

\begin{tabular}{|c|c|c|c|c|c|c|c|}
\hline $\mathcal{D C}$ & $\lambda$ & \multicolumn{7}{|c|}{ Buffer Size = 5 } \\
& & $(4,2)$ & $(5,3)$ & $(6,4)$ & $(7,5)$ & $(8,6)$ & $(9,7)$ \\
\hline \multirow{6}{*}{$25 \%$} & 1 & 3.8 & 1.5 & 0.7 & 0.3 & 0.14 & 0.08 \\
\cline { 2 - 8 } & 10 & 12.4 & 6.9 & 2.6 & 1.12 & 0.4 & 0.18 \\
\cline { 2 - 8 } & 20 & 15.3 & 7 & 3.4 & 1.5 & 0.8 & 0.32 \\
\cline { 2 - 8 } & 50 & 12.4 & 8.1 & 3.61 & 1.78 & 0.9 & 0.09 \\
\cline { 2 - 8 } & 1 & 3.65 & 1.18 & 0.52 & 0.37 & 0.096 & 0 \\
\cline { 2 - 8 } & 10 & 12.3 & 6.65 & 2.9 & 1.7 & 0.8 & 0.07 \\
\cline { 2 - 8 } & 20 & 13.4 & 7.4 & 4 & 1.7 & 0.8 & 0.5 \\
\cline { 2 - 8 } & 50 & 13.4 & 7.8 & 2.8 & 1.7 & 0.6 & 0.3 \\
\hline
\end{tabular}

Table III: \% Deferred Transmissions for each $(B O, S O)$. Duty Cycle $=25 \%$

\section{DISCUSSION}

We start by analysing those scenarios with low traffic where the arrival rate $\lambda=1$. Based on the packet drop rate we can characterize three types of scenarios: low traffic for those cases where the packet drop rate is zero and moderate and saturated traffic scenarios for those cases where packet drop rate is around $50 \%$ and above $80 \%$, respectively. As we 


\begin{tabular}{|c|c|c|c|c|c|c|c|}
\hline $\mathcal{D C}$ & $\lambda$ & \multicolumn{7}{|c|}{ Buffer Size = 5 } \\
& & $(3,2)$ & $(4,3)$ & $(5,4)$ & $(6,5)$ & $(7,6)$ & $(8,7)$ \\
\hline \multirow{6}{*}{$50 \%$} & 1 & 5.7 & 2.8 & 1.6 & 0.8 & 0.38 & 0.18 \\
\cline { 2 - 8 } & 10 & 13.1 & 6.3 & 2.4 & 1.2 & 0.6 & 0.26 \\
\cline { 2 - 8 } & 20 & 12.4 & 7.1 & 3.6 & 1.4 & 0.72 & 0.38 \\
\cline { 2 - 9 } & 50 & 12.9 & 7.7 & 3.6 & 1.5 & 0.85 & 0.47 \\
\cline { 2 - 8 } & 1 & 4.7 & 2.4 & 1.5 & 0.8 & 0.24 & 0.13 \\
\cline { 2 - 9 } & 10 & 11.4 & 6.3 & 3.27 & 1.79 & 0.66 & 0.25 \\
\cline { 2 - 9 } & 20 & 13.3 & 7.4 & 3.79 & 1.42 & 0.81 & 0.27 \\
\cline { 2 - 9 } & 50 & 13.3 & 8.82 & 3.83 & 1.4 & 0.72 & 0.3 \\
\hline
\end{tabular}

Table IV: \% Deferred Transmissions for each $(B O, S O)$. Duty Cycle $=50 \%$
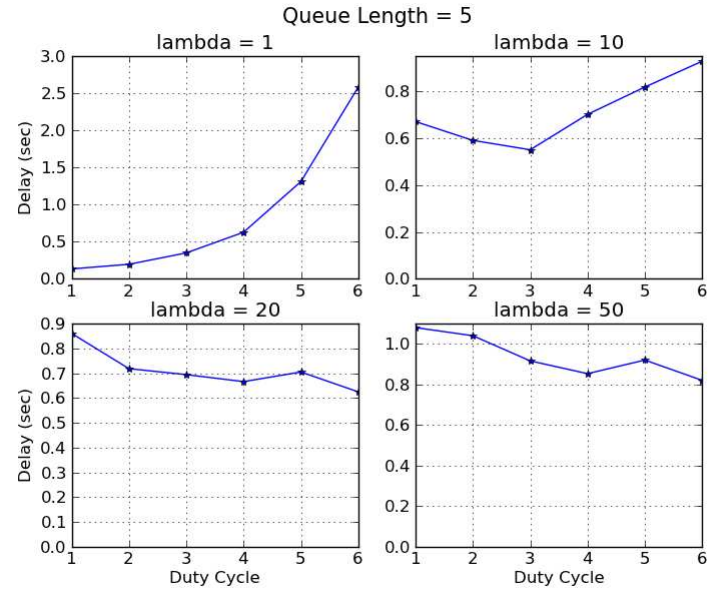

(a) Average Delay
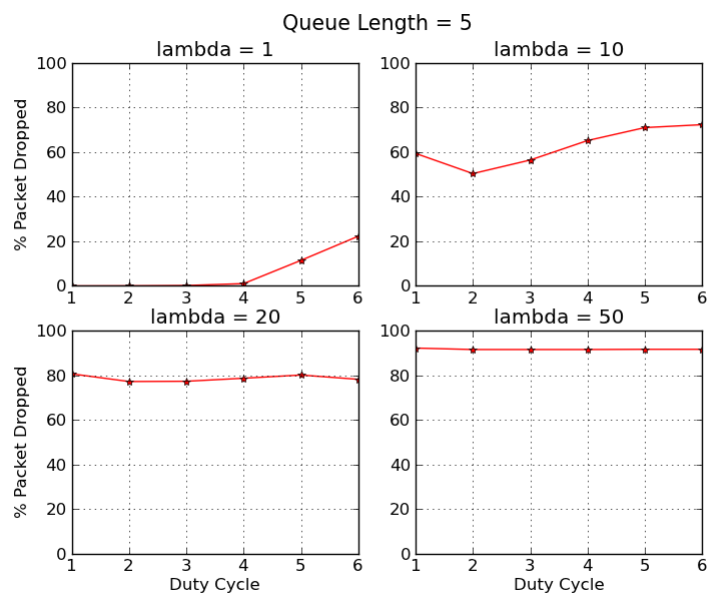

(b) Packet Drop

Figure 3: Duty Cycle $=25 \%$, Buffer Length $=5$.
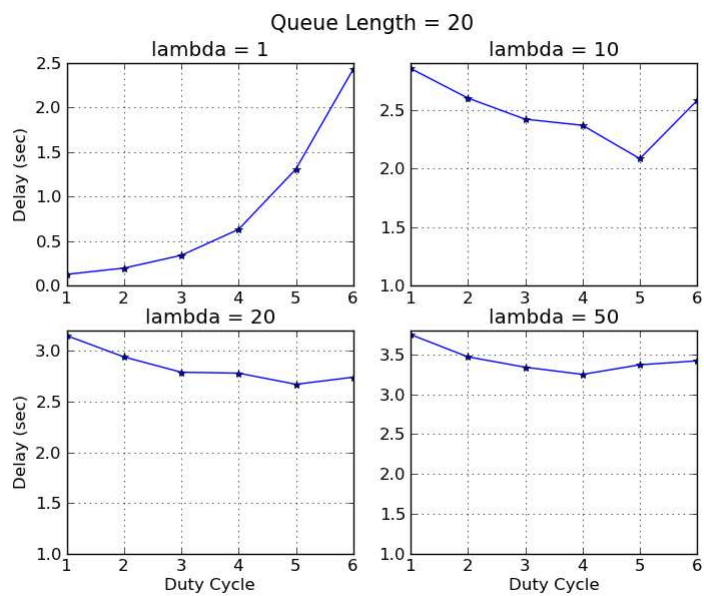

(a) Average Delay
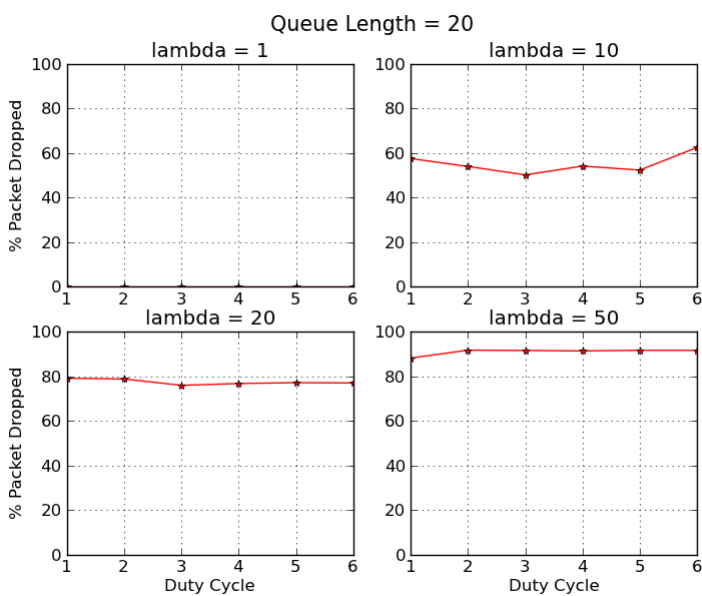

(b) Packet Drop

Figure 4: Duty Cycle $=25 \%$, Buffer Length $=20$.

can see from Figures 3, 4, 5 and 6, when $\lambda=1$ the packet drop rate is almost zero. Besides, arrival rate is very low for this scenario and then buffer in node is empty almost all the time. In these cases, delay for low traffic scenario increase exponentially when increasing $B O$ and $S O$. The reason is that, even that we keep the same duty cycle, increasing $B O$ and $S O$ gives a new superframe configuration where the inactive period is extended. That means the packet delay will increase for those arriving in the inactive period. Conversely, when $B O$ and $S O$ decrease, delay will decrease as well but in this case the number of beacon packets exchanged will increase and then the overall energy consumption. However, since buffer is almost empty and packet drop rate is almost 

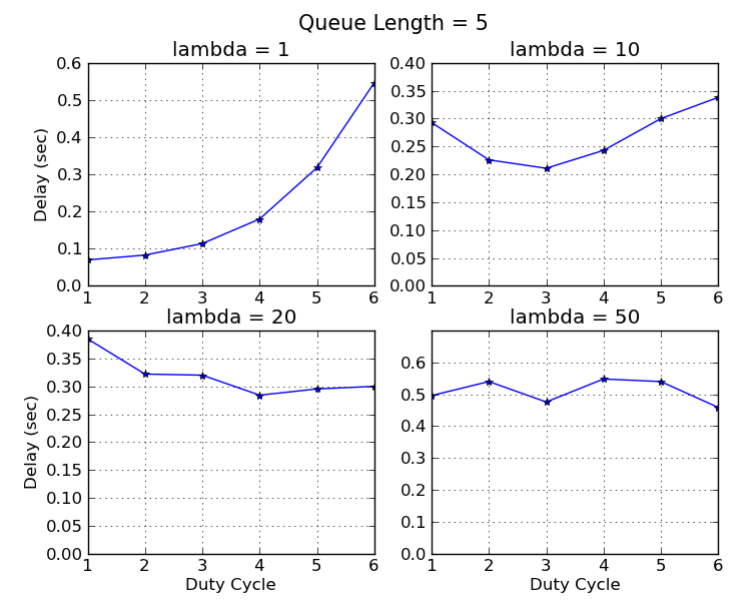

(a) Average Delay
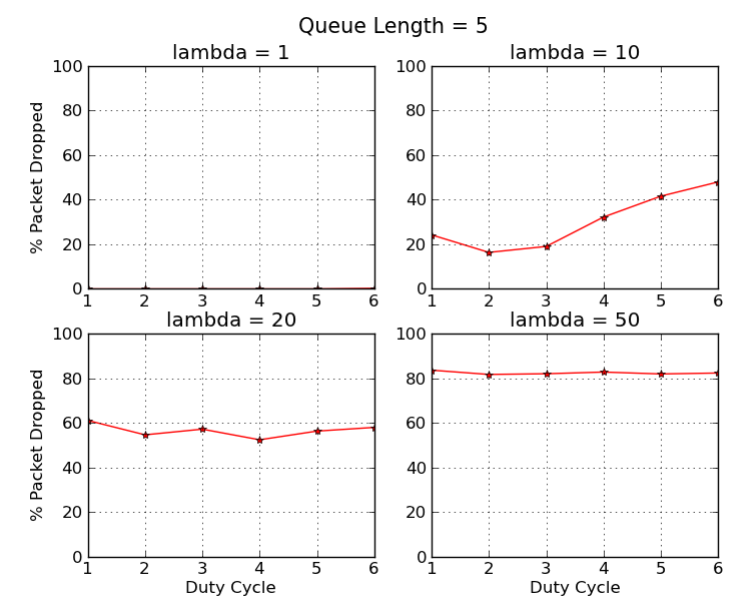

(b) Packet Drop

Figure 5: Duty Cycle $=50 \%$, Buffer Length $=5$.

zero, the throughput is not affected in this case. So for low traffic scenarios it should be necessary to find a trade-off between energy consumption and packet delay.

A more interesting result is obtained for almost saturated scenarios. When considering a $25 \%$ duty cycle and both $\lambda=20$ and $\lambda=50$, as well as considering a $50 \%$ duty cycle with arrival rate $\lambda=50$, we observe that packet drop rate is above $80 \%$ meaning that node buffer is full almost all the time. Looking at the corresponding delay graphics we can see that in almost all cases delay starts by decreasing while incrementing $B O$ and $S O$ until reaching certain point. After this point delay, either increases, as seen for both duty cycle boundaries configuration and buffer size $=20$ or fluctuates
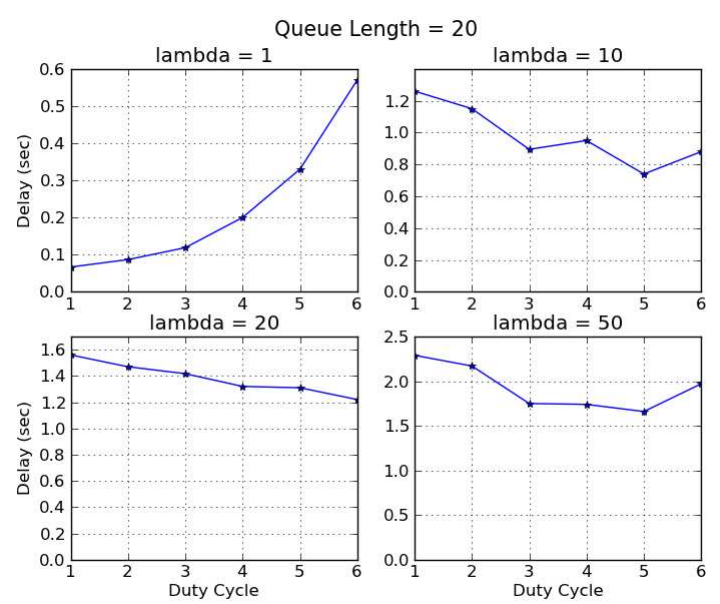

(a) Average Delay
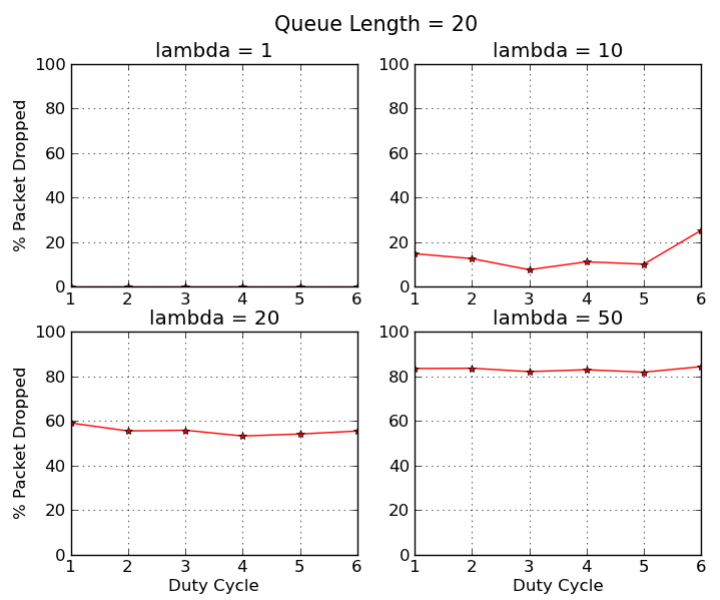

(b) Packet Drop

Figure 6: Duty Cycle $=50 \%$, Buffer Length $=20$.

between upper and lower boundaries not so far from each other, as seen for both duty cycles and buffer size $=5$. The only exception, where delay do not decrease but starts fluctuating from the beginning, is the case of 50\% duty cycle and buffer length $=5$. In order to understand this behavior we have to take into consideration another point that we have not mentioned before and is related to the number of transmissions deferred. On one hand, as we explained in section II, transmission is started if and only if the remaining number of backoff periods in the current superframe is sufficient to handle both the frame and the subsequent acknowledgement transmissions. Otherwise, transmission is deferred until the next superframe. On the other hand, as $(B O, S O)$ increases, 
the length of superframe also increases and then both active and inactive periods (always keeping the same $\mathcal{D C}$ ). When looking at the percentage of transmissions deferred for each of the $(B O, S O)$ configuration, we can see that the behavior is always the same independently of the scenario: percentage of deferred transmissions for $(B O+1, S O+1)$ is almost half the percentage of $(B O, S O)$. This is reasonable since a short superframe results in short active period and then the percentage of deferred transmission for short superframe is greater than the case of a long superframe. This issue will have an impact in the delay of those packets waiting in the buffer, specially in saturated scenarios where buffer is full almost all the time. As we increase the superframe length, we increment the length of the active period reducing the percentage of deferred transmission and then the average packet delay for those enqueued packets. However, increasing the superframe also increase both active and particularly the inactive period. After certain point, the length of the inactive period starts to impact the delay, as seen in most of the cases, incrementing the delay for those packets waiting in buffer as well as those packets arriving in the inactive period. In summary, delay is affected first due to a short length of the active period and percentage of deferred transmission and then, as we increment $(B O, S O)$, by the length of the inactive period. This behavior is not seen in low traffic scenarios since the buffer for these cases is empty almost all the time. Then, delay is affected only by the length of the inactive period and not by the deferred transmissions. Authors in [3] have shown that a large $B O$ increase the packet drop rate due to an extended inactive period. We can also see this behavior, specially for those scenarios with low traffic load as seen in Figure 3 for both $\lambda=1$ and 10, as well as in Figures 4, 5 and 6 for $\lambda=10$. On the other hand, for saturated scenarios the packet drop rate is not affected by the length of the inactive period but for the fact that buffer is full almost all the time.

As explained before, when both $B O$ and $S O$ decrease, the energy consumption increase due to the increment in the number of beacon packets exchanged. Additionally, energy consumption in saturated scenarios is also affected due to an increment in the channel contention activity at the start of the next superframe, since node's buffer is almost always full. In these scenarios, the number of drop packets remains almost constant so there is no significant impact in the throughput, that is to say, throughput is poor independently of the $(B O, S O)$ configuration. For saturated scenarios, since packet drop rate remains the same for all configurations, selecting the $(B O, S O)$ configuration that minimises the average delay seems to be the best option achieving also a reasonable energy consumption.

The same reasoning to explain why delay decrase to certain point and then increments or fluctuates can be applied for the cases of moderate traffic as seen in scenarios of $25 \%$ duty cycle, buffer length $=5$ and $\lambda=10$ as well as $50 \%$ duty cycle and $\lambda=20$ for both buffer size. For those scenarios packet drop rate is around $60 \%$. On the other hand, for moderate traffic scenarios, throughput is improved respected to saturated scenarios due to a lower rate of drop packets. Energy consumption is expected to be lower when comparing with saturated scenarios since the channel contention activity at the start of the next superframe decreases. Finally, a couple of results concerning those scenarios between low and moderate traffic as seen in $50 \%$ duty cycle and arrival rate $\lambda=10$. As we can see, delay behaves in the same way as saturated and moderate scenarios. However, packet drop rate starts decrasing until reaching the $(B O, S O)$ configuration that minimises the delay after which both packet drop rate and delay starts increasing. Selecting this configuration will give us an optimal solution in terms of both delay and packet drop rate keeping a moderate energy consumption. Finally, a question that may arise when looking at the high percentages of packets dropped. Why not considering a large buffer length in order to avoid having high rates of packets dropped ?. The answer is that we are working on Telosb motes which works with not enough memory so defining a large buffer would simply not be feasible.

\section{CONCLUSIONS \& Future WORK}

In this paper we have presented an analysis of the IEEE 802.15.4 duty cycle when considering a real scenario over TinyOS and Telosb motes. For several duty cycles we have studied different $(B O, S O)$ configurations and we have presented results in terms of the average delay from the moment a packet arrives to the MAC buffer until the corresponding acknowledgement is received, as well as the percentage of packets drop rate. For a given duty cycle $\mathcal{D} \mathcal{C}_{\#}$, we have analysed several $(B O, S O)$ configurations by changing the traffic arrival rate and node buffer length. We have seen that for scenarios having low traffic, average delay increases exponencially as $B O$ and $S O$ increase. In order to find the best configuration a trade-off between average delay and energy consumption must be taken into account since small $(B O, S O)$ introduces more overhead due to an increment in the beacon exchange. For saturated scenarios, we have shown that there is a $(B O, S O)$ configuration which minimises the delay keeping a reasonable energy consumption. Packet drop rate for these scenarios are poor since buffer node is almost all the time full. We have obtained the same conclusion also for the moderate scenarios except that in this case both energy consumption and packet drop rate were improved. Finally, for scenarios with traffic between low and moderate, an optimal $(B O, S O)$ configuration in terms of delay and packet drop rate was found. We were also able to verify the results obtained in [3] with respect to the packet drop rate. We conclude that, for low traffic scenarios, a large $B O$ increase the drop rate due to an extended inactive period. Based on the results and the discussion we have done we can conclude: 
a) That we are able to define a lower bound for the buffer length (LWBL) in order to avoid buffer overflow and thus avoiding the packet drops. This lower bound depends on both packet arrival rate and the length of the inactive period. In concrete, to avoid packet drops the following constraint must be satisfied:

$$
\text { LWBL }>\lambda * \text { LIP }
$$

where $\lambda$ is the arrival rate in packets per second and LIP is the length of the inactive period also in seconds. If buffer size is lower than LWBL then packets arriving in the inactive period will overflow the node buffer and then packets arriving right after will be dropped. This issue can be seen for the scenario with $\lambda=1$, buffer length $=5$ and $25 \%$ duty cycle where packet drop rate is zero for the first four $(B O, S O)$ configurations and non-zero for the last two configurations. For instance for the case $B O=9$ and $S O=7$, LIP is $5.9 \mathrm{~s}$ and then LWBL should be greater than this value in order to avoid dropping packets. Since the buffer length is 5 which is lower than LWBL then some packets are dropped. This condition is not a sufficient condition. At the beginning of the inactive period the buffer may not be empty and then packets arriving during this period may overflow the buffer and then packets coming right after will be dropped. However, it is the lowest buffer size that may guarantee no packet drops in the case that the buffer is empty when the inactive period starts.

b) There is a trade-off between energy consumption and delay that must be taken into account. Normally, when $(B O, S O)$ decreases the energy consumption increases due to an increment in the number of exchanged beacons. In scenarios with low traffic, choosing a small $(B O, S O)$ could be a good solution in terms of delay to the detriment of energy consumption. Conversely, by increasing $(B O, S O)$ we obtain a good solution in terms of energy consumption but not in terms of delay which increases exponencially. For the other scenarios, choosing a small $(B O, S O)$ seems not be a good solution, neither in terms of energy consumption nor in terms of average delay since as we saw, delay starts by decreasing until reaching an optimal point after which delay is not improved or even deteriorated. Selecting the optimal $(B O, S O)$ would be a good solution for these scenarios since we obtain an optimal average delay and a relative low energy consumption. We can then increase $(B O, S O)$ to have a better solution in terms of energy consumption to the detriment of the delay that increases or fluctuates between upper and lower boundaries not so far from each other.

As a future work and in order to avoid packet drops we plan to study an alternative where packets in buffer are overwritten by new packets arriving if buffer is full. This approach is useful for instance when data are measures such as temperature, humidity, etc. where we are only interested in getting the last measure. By overwriting the buffer we expect to reduce packet average delay.

\section{REFERENCES}

[1] IEEE Standard for Information Technology - Telecommunications and information exchange between systems - Local and metropolitan area networks - specific requirement Part 15.4: Wireless Medium Access Control (MAC) and Physical Layer (PHY) Specifications for Low-Rate Wireless Personal Area Networks (WPANs). Technical report, 2007.

[2] Francois Despaux, Ye-Qiong Song, and Abdelkader Lahmadi. On the gap between mathematical modeling and measurement analysis for performance evaluation of the 802.15 .4 mac protocol. In 12th International Workshop on Real Time Networks RTN13, 2013.

[3] Yu-Kai Huang, Ai-Chun Pang, and Hui-Nien Hung. A comprehensive analysis of low-power operation for beacon-enabled IEEE 802.15.4 wireless networks. IEEE Transactions on Wireless Communications, 8(11):5601-5611, November 2009.

[4] Mario Neugebauer, Jorn Plonnigs, and Klaus Kabitzsch. A new beacon order adaptation algorithm for IEEE 802.15. 4 networks. In Wireless Sensor Networks, 2005. Proceeedings of the Second European Workshop on, page 302311, 2005.

[5] Feng Shu, Taka Sakurai, Hai L. Vu, and Moshe Zukerman. Optimizing the IEEE 802.15. 4 MAC. In TENCON 2006. 2006 IEEE Region 10 Conference, page 14, 2006.

[6] M. Zuniga and B. Krishnamachari. Analyzing the transitional region in low power wireless links. In Sensor and Ad Hoc Communications and Networks, 2004. IEEE SECON 2004. 2004 First Annual IEEE Communications Society Conference on, page 517 526, 2004. 